\title{
An Investigation of the Pancreatic Islet Tumor Microenvironment with Time-of- Flight Secondary Ion Mass Spectrometry
}

\author{
Blake M. Bluestein ${ }^{1}$, Fionnuala Morrish ${ }^{2}$, David Hockenbery ${ }^{2}$, Lara J. Gamble ${ }^{1}$ \\ 1. Department of Bioengineering, NESAC/BIO, University of Washington, Seattle, WA, USA \\ 2. Fred Hutchinson Cancer Research Center, Seattle, WA, USA
}

Imaging time-of-flight secondary ion mass spectrometry (ToF-SIMS) provides molecular (chemical) information with subcellular spatial resolution. Here, imaging ToF-SIMS is used to analyze the tumor microenvironment in biopsies from a mouse model (Myc/p53-/-) with Myc-dependent inducible and regressible pancreatic $\beta$-cell neoplasia. The oncogene Myc is overexpressed in a wide variety of human cancers and strongly effects cellular metabolism, including lipid metabolism [1,2] It is hypothesized that chemical mapping of neoplastic tissues will reveal uptake and synthesis of fatty acids within the islet tumor induced by Myc. Lipid analysis via imaging ToF-SIMS will provide further insight into metabolism in the tumor microenvironment. While imaging ToF-SIMS analysis of tumor tissue will provide a new perspective by visualizing tumor progression/regression, the system itself can also act as a model system for investigating stroma-tumor interactions in cancerous tissues.

Pancreatic tissues were harvested and frozen in optimal cutting temperature (OCT) at 6 days post Myc induction. $4 \mu \mathrm{m}$ cryosections were cut, with one used for H\&E staining, one for ToF-SIMS analysis, and another for immunohistochemistry. High mass and high spatial resolution data was acquired with the pulsed $25 \mathrm{keV} \mathrm{Bi}_{3}{ }^{+}$ion beam rastered over a $1 \mathrm{~mm} \times 1 \mathrm{~mm}$ area $(1280 \times 1280$ pixels $)$. ROIs of the tumor and stromal tissue were then investigated further with imaging principal components analysis (PCA) to identify peaks that correspond to species of interest. Regions identified by analysis and PCA were then crossreferenced against immunohistochemical and $\mathrm{H} \& \mathrm{E}$ images to differentiate the tumor area from the surrounding tissue.

ToF-SIMS data suggests a preferential uptake of fatty acids 18:3, 18:2 and possibly phosphoinositol from lower mass molecular fragments in the negative polarity within the tumor. The 6 day Myc-induced islet tumor exhibits a signal of myristic acid (C14:0), possibly a product of de novo fatty acid synthesis within the tumor. The tumor also exhibits an increased localization of sphingomyelin fragments and vitamin $\mathrm{E}$ compared to the surrounding tissue. Interestingly, the data shows an absence of $\mathrm{Mg}^{+}$within the islet tumor (Figure 1C) and small, higher signal regions on the periphery of the tumor (Figures 1A and 2). These peripheral tumor regions exhibit an increased, localized signal of $\mathrm{CN}^{-}, \mathrm{CNO}^{-}, \mathrm{C}_{7} \mathrm{H}_{10} \mathrm{O}^{+}$, and $\mathrm{Fe}^{+}$. An ion image of $\mathrm{CN}^{-}$is shown in Figure 1A. Image PCA was applied to the entire image, illustrated in Figure 2 and reveals different chemistries within the tumor and surrounding acinar tissue. PCA was also applied to the selected tumor region images to spatially and chemically analyze within the tumor to compare different chemistries between different tumor sizes, which could indicate different tumor stage development, within the same tissue.

Image PCA separated the high signal regions at the periphery of tumors from the bulk of the tumors, but further histologic correlations are needed to discern if these structures are inflammatory zones, mitochondrial dense regions, or related to vasculature. Continuing work includes cell staining to identify these structures (work by our collaborators at the FHCRC). Once these localized areas have been better defined, a comparison of the regions to ToF-SIMS identified chemistry may aid in a valuable understanding of the Myc oncogene and its effect on pancreatic $\beta$-cell neoplasia. 


\section{References:}

[1]Nilsson, J.A. and J.L. Cleveland, Oncogene, 22, p. 9007.

[2]Morrish F. et al, J Biol Chem 285 (2010), p. 36267.

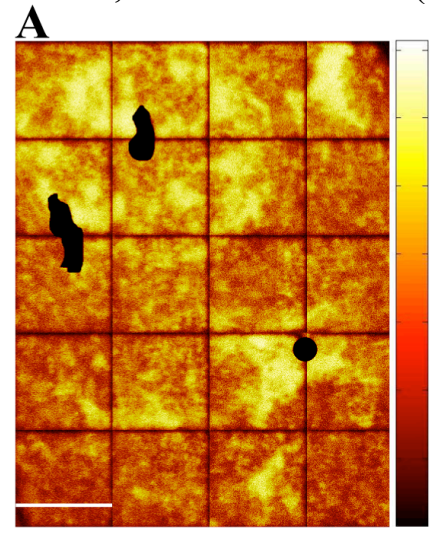

$\mathrm{CN}^{-}$

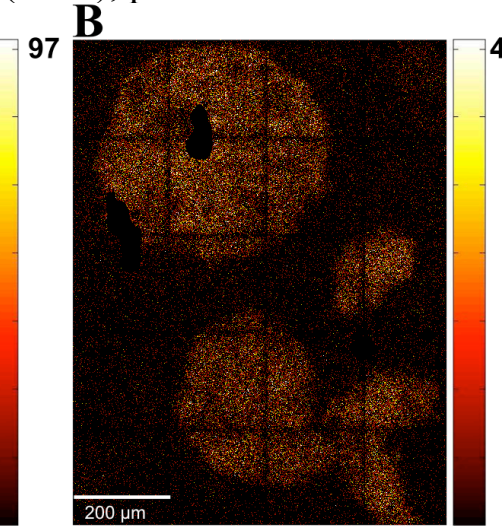

Myristic Acid

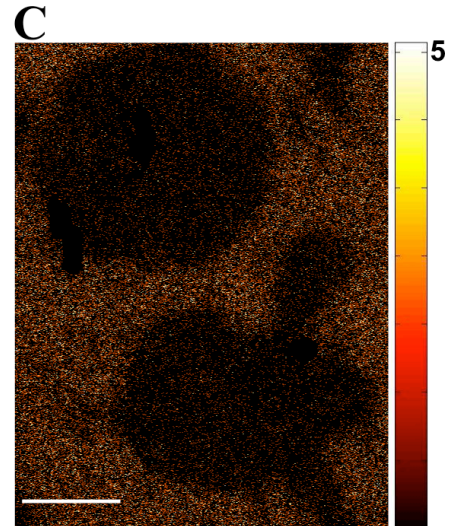

$\mathrm{Mg}^{+}$

C14:0

Figure 1. Ion images of two separate tumors. The ion image $\mathrm{CN}^{-}$in $\mathrm{A}$ indicates protein rich regions within the tumor defined area, B shows the tumor regions rich with a fully saturated fatty acid, C14:0 and the ion image $\mathrm{C}$ displays the absence of $\mathrm{Mg}^{+}$within the tumors. All images were binned by a factor of 4 pixels to increase contrast for this figure.

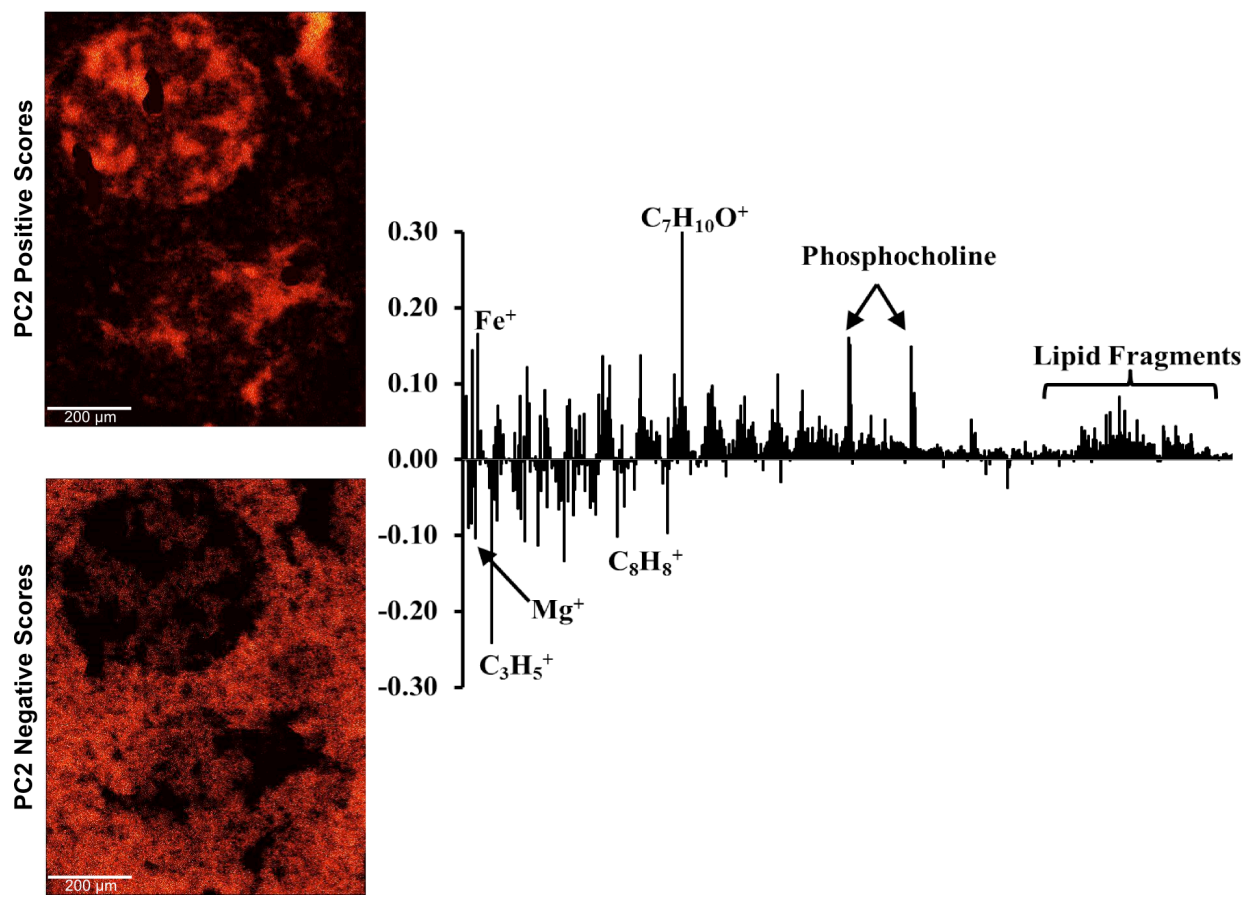

Figure 2. Image PCA distinctly separates the tumors from the surrounding tissue. The positive scores (top, left image) corresponds to the positive loadings (graph at right, above $\mathrm{y}=0$ ), where $\mathrm{Fe}^{+}$, protein rich regions, and lipid fragments separate the tumor from the outer tissue. Negative scores (bottom, left image) correspond to negative loadings (graph at right, below $\mathrm{y}=0$ ) where $\mathrm{Mg}^{+}$and hydrocarbon fragmented regions are observed in the tissue surrounding the tumor. 